Schmerz 2023 · 37:123-133

https://doi.org/10.1007/s00482-022-00624-2

Eingegangen: 13. April 2021

Überarbeitet: 17. Dezember 2021

Angenommen: 4. Januar 2022

Online publiziert: 22. Februar 2022

(c) Der/die Autor(en) 2022

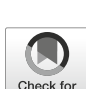

check for
updates

\section{Weniger Operationen und Kosten wegen Rückenschmerzen in einem Versorgungsprogramm mit interdisziplinärem Zweitmeinungsverfahren}

\author{
Eine kontrollierte, nichtrandomisierte Interventionsstudie \\ mit GKV-Daten
}

Christoph J. Wagner' - Gabriele Lindena ${ }^{2} \cdot$ Grit Ayyad $^{1} \cdot$ Andrea Otzdorff' - Ulf Marnitz ${ }^{3}$. Karen Bienek ${ }^{4}$. Björn von Pickardt ${ }^{5}$. Stephanie Sehlen ${ }^{1}$. Werner Wyrwich ${ }^{1}$

${ }^{1}$ AOK Nordost, Berlin, Deutschland

${ }^{2}$ CLARA Clinical Analysis Research and Application, Kleinmachnow, Deutschland

${ }^{3}$ Rückenzentrum am Markgrafenpark GmbH, Berlin, Deutschland

${ }^{4}$ Reha-Tagesklinik im Forum Pankow GmbH und Co. KG, Berlin, Deutschland

${ }^{5}$ Reha-Zentrum Teltow GmbH und Co. KG, Teltow, Deutschland
Zusatzmaterial online

Die Online-Version dieses Beitrags (https:// doi.org/10.1007/s00482-022-00624-2) enthält weitere Tabellen.

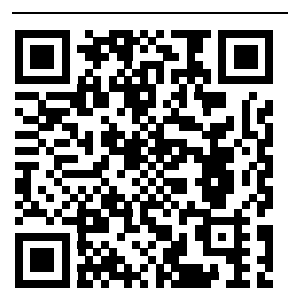

QR-Code scannen \& Beitrag online lesen

\section{Zusammenfassung}

Hintergrund: Für interdisziplinäre Zweitmeinungsverfahren (IZMV) bei empfohlenen Rückenoperationen (ROP) fehlen Wirksamkeitsbelege. Die AOK Nordost bietet seit 2015 das Versorgungsprogramm RückenSPEZIAL an. Es enthält Vorbefundprüfung, IZMV und optional eine interdisziplinär-multimodale Schmerztherapie (IMST). Ziel dieser Studie ist die Ermittlung der Wirksamkeit von RückenSPEZIAL für ROP und rückenschmerzbezogene Kosten (RBK) im Vergleich zu Patienten mit ROP-Empfehlung ohne RückenSPEZIAL.

Methoden: Versicherte der AOK Nordost legten im AOK-Servicecenter einen ROPKrankenhauseinweisungsschein vor und erhielten eine dokumentierte Beratung zur RückenSPEZIAL-Teilnahme. Nach einer 1:1-Matched-pairs-Ziehung wurden Patienten, die nach dieser Beratung (=Stichtag) an RückenSPEZIAL teilnahmen (Interventionsgruppe [IG]), mit Patienten verglichen, die nach der Beratung nicht teilnahmen (Vergleichsgruppe [VG]). Patientenmerkmale, ROP und RBK wurden aus Daten der AOK Nordost operationalisiert.

Ergebnisse: Von 108 IG-Patienten und 108 VG-Patienten hatten 34 (42\%) weniger IGPatienten eine oder mehr ROP in 365 Folgetagen (relatives Risiko [RR] 0,58; $p<0,001$ ). Die Subgruppenanalyse zeigte für 21 IG-Patienten mit IZMV und IMST ein RR von 0,13 $(p<0,001)$ und für 67 IG-Patienten mit alleinigem IZMV ohne IMST ein RR von 0,59 $(p<0,001)$. Die Veränderung der RBK von Vorjahr auf Folgejahr war für die IG um $50,2 \%$-Punkte geringer als für die VG $(p=0,088)$.

Diskussion: Die ROP-Unterschiede zugunsten des Selektivvertrags RückenSPEZIAL waren signifikant $(p<0,05)$. Für die spezifische Population ist eine Refinanzierung der Interventionskosten von RückenSPEZIAL tendenziell erwartbar (näherungsweise signifikant, geringe Fallzahl). Es ist eine Verzerrung aufgrund von Selbstselektion anzunehmen.

\section{Schlüsselwörter}

Planbare Operation · Wirbelsäulenoperation · Chronischer Rückenschmerz · Schmerztherapie Krankheitskosten 


\section{Einleitung}

Versicherte der gesetzlichen Krankenversicherung (GKV) haben seit dem GKVVersorgungsstärkungsgesetz 2015 in Deutschland einen Anspruch auf ärztliche Zweitmeinung vor bestimmten planbaren Operationen ( $§ 27 b$ SGB V). Eingriffe an der Wirbelsäule wurden allerdings erst mit Inkrafttreten zum 19.11.2021 in die Liste der anspruchsberechtigenden Operationen aufgenommen [5]. Seit 2015 nutzen daher Krankenkassen wie die AOK Nordost Verträge zur besonderen Versorgung (§§ 140 SGB V), um Ihren Versicherten selbst konzipierte Zweitmeinungsverfahren bei Rückenoperationen (ROP) anzubieten und diese auch zu evaluieren.

In 2018 gab es in Deutschland 815.295 ROP-Krankenhausfälle mit Operationenund Prozedurenschlüssel (OPS) Kapitel 583 [2]. In 2018 kostete ein solcher ROPKrankenhausfall bei der AOK Nordost durchschnittlich $8960 €$. Es gibt Hinweise, dass ROP teils keinen Nutzen generieren und teils aufgrund wirtschaftlicher Anreize des Krankenhaus-Vergütungssystems durchgeführt werden [8].

Für ROP fehlen bislang kontrollierte Studien zu Zweitmeinungsverfahren, die in einer Nachbeobachtung die tatsächlich erfolgten Rückenoperationen (ROP) sowie die rückenschmerzbezogenen Kosten (RBK) mit einer Vergleichsgruppe analysieren $[1,9,11]$. Die Information zu einer ROP-Erstempfehlung liegt in der Regel nicht systematisch in den Abrechnungsdaten der GKV vor. In dieser Studie gelang es, ROP-Erstempfehlungen systematisch über ärztliche Krankenhauseinweisungsscheine zu identifizieren, die der AOK Nordost vorgelegt wurden. Diese Informationsbasis ermöglichte einen Matched-

\section{Abkürzungen}

\begin{tabular}{ll} 
GKV & $\begin{array}{l}\text { Gesetzliche Krankenversicherung } \\
\text { Interventionsgruppe }\end{array}$ \\
IMST & $\begin{array}{l}\text { Interdisziplinär-multimodale } \\
\text { Schmerztherapie }\end{array}$ \\
IZMV & $\begin{array}{l}\text { Interdisziplinäres Zweitmeinungs- } \\
\text { verfahren }\end{array}$ \\
RBK & Rückenschmerzbezogene Kosten \\
ROP/OP & Rückenoperation \\
$V G$ & Vergleichsgruppe \\
\hline
\end{tabular}

pairs-Vergleich von Versicherten mit einer ROP-Erstempfehlung "mit" versus "ohne" besondere Versorgung mit einem Zweitmeinungsverfahren.

Die AOK Nordost bietet ihren Versicherten seit dem 15.04.2015 das Versorgungsprogramm RückenSPEZIAL als Vertrag über eine besondere Versorgung nach $\S \S 140$ a ff. SGB V an. Versicherte mit Krankenhauseinweisungsschein für eine ROP können teilnehmen und erhalten in 5 ambulanten Rückenzentren in Berlin und Brandenburg eine Vorbefundprüfung, ein interdisziplinäres Zweitmeinungsverfahren (IZMV) sowie auf Empfehlung optional eine interdisziplinär-multimodale Schmerztherapie (IMST). In der Vorbefundprüfung durch den Arzt werden fakultative Ausschlusskriterien zur Orientierung geprüft (siehe - Tab. 2). Das IZMV ist zentrale Vertragsleistung. Es enthält ein ca. dreistündiges Assessment mit ärztlicher Diagnostik, schmerzpsychologischem Screening und physiotherapeutischer Befunderhebung, eine interdisziplinäre Fallkonferenz mit Einbindung/Kontaktierung des Erstmeiners, ein Versichertengespräch mit individueller Therapieempfehlung sowie die Arztbrieferstellung. Auf Empfehlung des IZMV hin ergeben sich drei Subgruppen der Weiterbehandlung nach dem IZMV (- Abb. 1):

1. Der Patient erhält auf ärztliche Empfehlung hin die zusätzliche RückenSPEZIAL-Leistung IMST (,IZMV und IMST").

2. Der Patient wird ohne weitere RückenSPEZIAL-Leistungen mit einem Arztbrief, Empfehlungen und oftmals einem Telefonat mit dem Erstmeiner zurück in die Therapie der ambulanten Regelversorgung entlassen („IZMV und Regelversorgung").

3. Der Patient erhält eine direkte OPEmpfehlung (,IZMV und direkte OPEmpfehlung").

Im Rahmen der IMST [7] erbringen in einem ambulanten Versorgungssetting Fachärzte für Orthopädie, physikalische und Rehabilitationsmedizin, Anästhesiologie, Ärzte mit Zusatzqualifikation Schmerztherapeut, Psychotherapeuten, Sozialtherapeuten, Physiotherapeuten, Sporttherapeuten, Ergotherapeuten je nach Versichertensituation drei mögliche Leistungspakete: (i) Vollzeitteilnahme für $100 \mathrm{~h}$ in 20 Tagen, (ii) Vollzeitteilnahme für $60 \mathrm{~h}$ in $\leq 4$ Wochen, (iii) berufsbegleitende Teilnahme für $40-50 \mathrm{~h}$ in $\leq 3$ Monaten. Die IMST umfasst als ambulante modulare Gruppentherapie die Komponenten Schmerztherapie, Edukation, Psycho-, medizinische Trainings- und Ergotherapie sowie regelmäßige interdisziplinäre Fallkonferenzen.

Ziel dieser Studie ist es, die Wirksamkeit des Vertrags RückenSPEZIAL hinsichtlich einer Reduktion der ROP und RBK im Folgejahr zu analysieren. Verglichen wird mit Patienten, die gleichermaßen eine ärztliche Erstmeinung für eine ROP, aber keine nachfolgende Zweitmeinung und ggf. IMST erhalten haben.

\section{Methoden}

\section{Daten, Studienpopulation und -design}

Es wurde retrospektiv eine kontrollierte, nichtrandomisierte Interventionsstudie auf Basis von Versichertenstammdaten, Leistungsabrechnungsdaten der AOK Nordost sowie Daten des Fallmanagementsystems der AOK-Servicecenter durchgeführt. Im Fallmanagementsystem der AOK-Servicecenter werden Versichertenberatungen dokumentiert. Darin wurde erfasst, ob ein Krankenhauseinweisungsschein für eine ROP im AOKServicecenter vorgelegt wurde und ob im Zuge dieser Vorlage eine Empfehlung zur RückenSPEZIAL-Teilnahme durch die AOK Nordost erfolgte. Damit war es möglich, eine Grundgesamtheit von Versicherten zu identifizieren, die im Zeitraum vom 05.02.2015 bis zum 30.03.2017 die zwei zentralen Aufgreifkriterien erfüllte:

- Ärztliche Erstmeinung dokumentiert per ROP-Krankenhauseinweisungsschein,

- Beratungsgespräch zur Teilnahme an RückenSPEZIAL (• Abb. 1).

Die AOK-Servicecenter-Mitarbeiter prüften vor einer Beratung, ob eine augenscheinlich klar erkennbare Nichteignung für das Zweitmeinungsverfahren vorlag. Versicherte, die binnen 74 Tagen nach der RückenSPEZIAL-Teilnahmebera- 


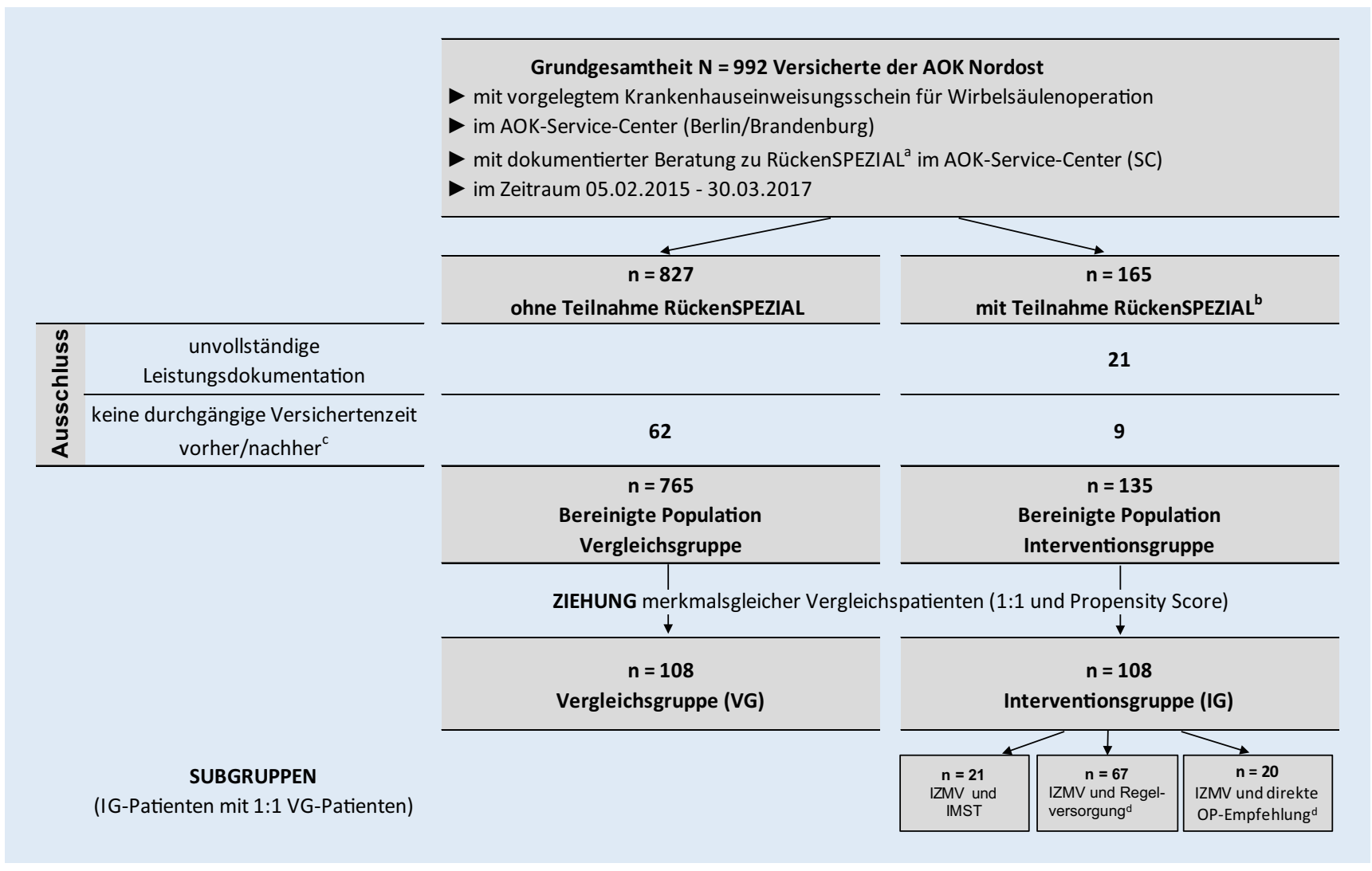

Abb. 1 ム Identifikation von Interventionsgruppe und Vergleichsgruppe. IZMV interdisziplinäres Zweitmeinungsverfahren;

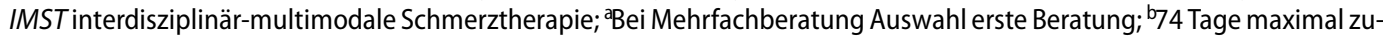
lässiger Zeitabstand zwischen SC-Beratung und Erstkontakt Rückenzentrum; 8 Quartale vor und 4 Quartale nach Indexquartal, hier ebenfalls bereinigt:4 Patienten im Pflegeheim sowie 3 Patienten ohne vollständige Stammdatendokumentation oder Versichertenart; ' Ein Patient dieser Gruppe erhielt lediglich „Vorbefundprüfung" ohne IZMV

tung mindestens die "Vorbefundprüfung" von RückenSPEZIAL in Anspruch genommen hatten, gehörten zur Population mit Teilnahme RückenSPEZIAL. Diese Population stellte die Basis für die Interventionsgruppe (IG) dar. Versicherte, die nach der RückenSPEZIAL-Teilnahmeberatung keine RückenSPEZIAL-Leistung in Anspruch genommen hatten, gehörten zur Population ohne Teilnahme am Vertrag RückenSPEZIAL. Aus dieser Population wurde im Weiteren die Vergleichsgruppe (VG) gebildet. 74 Tage wurden als Obergrenze für eine beratungsbezogene Teilnahme verwendet, da $95 \%$ aller Tagesdifferenzen zwischen RückenSPEZIAL-Teilnahmeberatung und Erstkontakt (Vorbefundprüfung und/oder IZMV) bei RückenSPEZIAL kleiner gleich 74 Tage waren.

Der Versichertenkontakttag im AOKServicecenter war der Stichtag für die Wirksamkeitsbeobachtung in 365 Folgetagen sowie für den Merkmalsabgleich der Vergleichsgruppenziehung in 365 oder 730 Tagen vorab. Patienten ohne durchgängige Versichertenzeit in $730 \mathrm{Ta}$ gen vor und 365 Tagen nach Stichtag wurden ausgeschlossen. Für sie waren Vergleichsmerkmale oder Nachbeobachtungszeit nicht vollständig verfügbar. Ebenso mussten 21 RückenSPEZIAL-Teilnehmer ausgeschlossen werden, deren Leistungsdokumentationen aus organisatorischen Gründen nicht vorlagen. Aus diesen bereinigten Populationen von IG und VG wurden in zwei Ziehungsschritten mit exaktem "matching" und "propensity score matching" merkmalsgleiche Vergleichspatientenpaare im Verhältnis 1:1 ausgewählt ohne Zurücklegen. Die Analyse befolgte Standards für Sekundärdatenanalyse und Beobachtungsstudien [12, 14].

\section{Ziehung merkmalsgleicher Vergleichspatienten}

Im Ziehungsschritt 1 wurde für zentrale, vor allem rückenschmerzbezogene Merkmale ein exaktes "1:1 matching" durchgeführt. Je Versicherten aus der bereinigten Population der IG wurde aus der bereinigten Population der VG ein bestgeeigneter Matching-Partner gesucht, der für neun definierte Merkmale eine identische Merkmalskombination aufwies (•Tab. 2, Merkmalsabgleich, "1:1", kursiv hervorgehobene Zeilen): Das Alter wurde mit einer Spannweite von \pm 10 Jahre abgeglichen. Einzelkategorien der Merkmale "Bundesland“, „Dauer kontinuierliche quartalsweise ambulante Arztkontakte/Krankenhausaufenthalte mit Rückenschmerzdiagnose" sowie „Rückenschmerzdiagnose" wurden teils gemäß Verteilung zusammengefasst, um eine hinreichende Treffermenge zu ermöglichen ( $\mathbf{0}$ Tab. 2). Weitere Variablen wurden 
Tab. 1 Operationalisierung rückenschmerzbezogener Kosten und Leistungsmerkmale

\begin{tabular}{|l|l|}
\hline Leistung/Merkmal & Operationalisierung \\
\hline Rückenoperation & $\begin{array}{l}\text { Krankenhausfälle oder Fälle von ambulantem Operieren mit stationärem Aufnahmedatum oder ambulantem Behandlungs- } \\
\text { datum im Beobachtungszeitraum und mit OPS-Code-Gruppe 5-83 für Wirbelsäulenoperationen sowie weiteren OPS-Codes } \\
\text { für Flavektomie, Laminotomie, Hemilaminektomie und Laminektomie }\end{array}$ \\
\hline $\begin{array}{l}\text { Krankenhausfall ohne } \\
\text { Rückenoperation }\end{array}$ & $\begin{array}{l}\text { Krankenhausfälle mit Aufnahmedatum im Beobachtungszeitraum und mit definierten ICD-10-Codes für Rückenschmerz als } \\
\text { Hauptentlassdiagnose ohne einen OPS-Code für Rückenoperation }\end{array}$ \\
\hline Schmerzarzneimittel & Nettokosten für definierte ATC-Codes mit Verordnungsdatum im Beobachtungszeitraum \\
\hline Physiotherapie & $\begin{array}{l}\text { Alle Verordnungen mit dokumentiertem Indikationsgebiet Wirbelsäulenerkrankungen 1 und Wirbelsäulenerkrankungen 2 } \\
\text { des Heilmittelkatalogs nach § 92 Abs. 6 Satz 1 Nr. 2 SGB V mit Behandlungsdatum im Beobachtungszeitraum }\end{array}$ \\
\hline $\begin{array}{l}\text { Ambulante Facharzt- } \\
\text { behandlung }\end{array}$ & $\begin{array}{l}\text { Die gesamten Fallkosten für alle Behandlungsfälle mit mindestens einem Behandlungstag des Behandlungsfalls im Beob- } \\
\text { achtungszeitraum, bei den Arztgruppen Orthopäde, Radiologe, physikalische und rehabilitative Medizin, Neurochirurgie, } \\
\text { Neurologie, Neurologie/Psychiatrie, wenn mindestens eine gesicherte Rückenschmerzdiagnose codiert wurde }\end{array}$ \\
\hline Hilfsmittel & $\begin{array}{l}\text { Ausgewählte Gebührenpositionen der Produktgruppen Bandagen, Einlagen, Elektrostimulationsgeräte, Orthesen/ } \\
\text { Schienen, Schuhe mit Leistungsbeginndatum im Beobachtungszeitraum }\end{array}$ \\
\hline $\begin{array}{l}\text { Stationäre Rehabilita- } \\
\text { tion }\end{array}$ & $\begin{array}{l}\text { Fallkosten der Behandlungsfälle mit Hauptdiagnose oder Operationsdiagnose Rückenschmerz mit Beginndatum im Beob- } \\
\text { achtungszeitraum }\end{array}$ \\
\hline $\begin{array}{l}\text { Ambulante Rehabili- } \\
\text { tation }\end{array}$ & $\begin{array}{l}\text { Leistungen zu Rehabilitationssport/Funktionstraining gemäß Reha-Leistungs-Schlüssel mit Leistungsbeginn im Beobach- } \\
\text { tungszeitraum }\end{array}$ \\
\hline Krankengeld & $\begin{array}{l}\text { Alle Krankengeldfälle mit Beginndatum im Beobachtungszeitraum mit definierten ICD-10-Codes für Rückenschmerz. Kran- } \\
\text { kengeldzahlungen sind einkommensabhängig. Zur Vergleichbarkeit wurden für Interventionsgruppe und Vergleichsgrup- } \\
\text { pe durchschnittliche Krankengeldtageskosten der Interventionsgruppe verwendet (Standardisierung) }\end{array}$ \\
\hline
\end{tabular}

dichotom operationalisiert. Mindestens einmaliges Vorkommen des Merkmals regelhaft im Vorjahr bedeutet "1“. Keinerlei Vorkommen des Merkmals regelhaft im Vorjahr bedeutet "0". Im Ergebnis von Ziehungsschritt 1 gab es IG-Patienten, für die mehrere geeignete VG-Patienten vorlagen. Für diese erfolgte Ziehungsschritt 2.

Für Ziehungsschritt 2 wurden weitere Einflussgrößen als "propensity score" zusammengefasst. Zur Ermittlung des „propensity score“" wurde mit logistischer Regression für die bereinigten Populationen von IG und VG die Wahrscheinlichkeit geschätzt, zur IG zu gehören, anhand einer erweiterten Liste von Merkmalen für rückenschmerzbezogene Leistungen, psychische Begleiterkrankungen, generelle Krankenhausaufenthalte sowie die Anzahl unterschiedlicher Arzneimittelwirkstoffe (- Tab. 2, Merkmalsabgleich, „PS“). Danach wurde für jeden IG-Patienten mit mehreren potenziellen 1:1-VG-Patienten aus Ziehungsschritt 1 derjenige mit dem ähnlichsten „propensity score“ ausgewählt (Nearest-neighbour-Methode).

Für die resultierenden IG-Patienten mit ihren jeweiligen individuell merkmalsgleichen VG-Patienten erfolgte ein Ergebnisvergleich in der Gesamtgruppe sowie in drei für die Evaluation definierten Subgruppen gemäß IZMV-Empfehlung: (i) IZMV und IMST, (ii) IZMV und Regel- versorgung, (iii) IZMV und direkte OPEmpfehlung (• Abb. 1).

\section{Rückenschmerzbezogene Kosten und Merkmale}

Folgende rückenschmerzbezogene Kosten (RBK) wurden im Beobachtungszeitraum von 365 Tagen ab Stichtag bestimmt: Kosten für stationäre/ambulante Rückenoperationen, Krankenhausaufenthalte mit Hauptentlassdiagnose Rückenschmerz ohne ROP, Schmerzarzneimittel, Physiotherapie, ambulante Facharztbehandlung, Hilfsmittel, ambulante und stationäre Rehabilitation und Krankengeldzahlung. Die Operationalisierung der Kosten und Leistungen wird in $\bullet$ Tab. 1 beschrieben. Diese Operationalisierung wurde auch für das „matching" verwendet für den Zeitraum vor dem Stichtag. Für Versicherte der IG wurden zudem die zusätzlichen Interventionskosten des Vertrags RückenSPEZIAL berechnet.

Für alle so definierten Leistungen wurde der Zahlbetrag der GKV analysiert ohne etwaige Patientenzuzahlungen (GKV-Perspektive). Der Anstieg der RBK vom Vorjahr auf das Folgejahr in der IG - einschließlich der zusätzlichen Interventionskosten des Vertrags RückenSPEZIAL - wurde mit dem RBK-Anstieg in der VG verglichen. Diese Differenz-von-Differenzen-Methode wurde verwendet, um für Kostenunterschiede im Ausgangsniveau im Vorjahr zu kontrollieren. Aus Gründen der Geheimhaltung wurden Kostenergebnisse lediglich prozentual veranschaulicht.

Das Online-Zusatzmaterial listet eine vollständige Übersicht der verwendeten ICD-10, OPS-Codes etc. für Kosten- und Matching-Variablen.

\section{Statistische Analyse}

Häufigkeitsunterschiede von Gruppenmerkmalen wurden mittels T-Test für unverbundene Stichproben oder $\mathrm{X}^{2}$-Test beschrieben. Häufigkeitsunterschiede der Ergebnisvariable „Anzahl Patienten mit mindestens einer ROP in 365 Folgetagen" zwischen IG und VG wurden als relatives Risiko (RR) mit Konfidenzintervallen basierend auf Normalverteilungsannahme veranschaulicht.

Aufgrund der sehr uneinheitlich verteilten teuren ROP-Ereignisse und wegen der pauschalierten Kostenabrechnung für die Leistungen des Vertrags RückenSPEZIAL bei den IG-Patienten war eine Approximation einer Verteilung der RBK nicht möglich. Daher wurde eine Bewertung mittels Bootstrapping-Verfahren und 10.000 Iterationen der Stichprobe durchgeführt. Mit der stichprobenbasierten Verteilung und einem einseitigen Test wurde die gerich- 
Tab. 2 Beschreibung der Vergleichsmerkmale vor oder bei Beobachtungsbeginn

\begin{tabular}{|c|c|c|c|c|c|c|c|}
\hline \multirow[b]{2}{*}{ Merkmale } & \multicolumn{2}{|c|}{$\begin{array}{l}\text { Interventionsgruppe } \\
(I G, N=108)\end{array}$} & \multicolumn{2}{|c|}{$\begin{array}{l}\text { Vergleichsgruppe } \\
\text { (VG, } N=108 \text { ) }\end{array}$} & \multirow{2}{*}{ 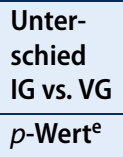 } & \multicolumn{2}{|l|}{ Merkmalsabgleich } \\
\hline & $n$ & $\%$ & $n$ & $\%$ & & Ziehungsschritt 1 & Ziehungsschritt 2 \\
\hline \multicolumn{8}{|l|}{ Soziodemografie $^{a}$} \\
\hline Alter $\varnothing(S D)$ [Median] & \multicolumn{2}{|c|}{$61,3(13,1)[61]$} & \multicolumn{2}{|c|}{$61,1(12,6)[61]$} & 0,90 & $1: 1$ & $P S$ \\
\hline Weiblich & 60 & $(55,6)$ & 60 & $(55,6)$ & 1,00 & $1: 1$ & $P S$ \\
\hline Pflegestufe ja/nein & 3 & $(2,8)$ & 3 & $(2,8)$ & 1,00 & $1: 1$ & $P S$ \\
\hline \multicolumn{8}{|l|}{ Wohnort $^{\mathrm{a}}$} \\
\hline Berlin & 78 & $(72,2)$ & 78 & $(72,2)$ & 1,00 & $1: 1$ & $P S$ \\
\hline Brandenburg & 30 & $(27,8)$ & 30 & $(27,8)$ & 1,00 & $1: 1$ & $P S$ \\
\hline \multicolumn{8}{|l|}{ Versicherungsart $^{a}$} \\
\hline Familienmitversichert & 4 & $(3,7)$ & 3 & $(2,8)$ & n.a. & - & PS \\
\hline Pflichtversichert & 25 & $(23,1)$ & 28 & $(25,9)$ & 0,68 & - & PS \\
\hline Rentner & 51 & $(47,2)$ & 51 & $(47,2)$ & 1,00 & - & PS \\
\hline Arbeitslos & 19 & $(17,6)$ & 18 & $(16,7)$ & 0,87 & - & PS \\
\hline Freiwillig & 6 & $(5,6)$ & 7 & $(6,5)$ & 0,78 & - & PS \\
\hline Sonstiges & 3 & $(2,8)$ & 1 & $(0,9)$ & n.a. & - & PS \\
\hline \multicolumn{8}{|c|}{ Dauer kontinuierlicher Rückenschmerzdiagnosen in 2 Vorjahren ${ }^{c}$} \\
\hline OQuartale & 6 & $(5,6)$ & 6 & $(5,6)$ & 1,00 & $1: 1$ & $P S$ \\
\hline 1 Quartal & 14 & $(13,0)$ & 15 & $(13,9)$ & 0,85 & \multirow[t]{7}{*}{$1: 1^{f}$} & $P S$ \\
\hline 2 Quartale & 9 & $(8,3)$ & 10 & $(9,3)$ & 0,82 & & $P S$ \\
\hline 3 Quartale & 10 & $(9,3)$ & 7 & $(6,5)$ & 0,47 & & PS \\
\hline 4 Quartale & 4 & $(3,7)$ & 5 & $(4,6)$ & n. $a$. & & $P S$ \\
\hline 5 Quartale & 4 & $(3,7)$ & 2 & $(1,9)$ & n.a. & & $P S$ \\
\hline 6 Quartale & 0 & $(0,0)$ & 2 & $(1,9)$ & n.a. & & PS \\
\hline 7 Quartale & 3 & $(2,8)$ & 3 & $(2,8)$ & n.a. & & $P S$ \\
\hline 8 Quartale & 58 & $(53,7)$ & 58 & $(53,7)$ & 1,00 & $1: 1$ & $P S$ \\
\hline \multicolumn{8}{|l|}{ Rückenschmerzdiagnosegruppe 1 im Vorjahr ${ }^{b, c}$} \\
\hline Lumbal \& zervikald & 61 & $(56,5)$ & 61 & $(56,5)$ & 1,00 & $1: 1$ & PS \\
\hline Zervikal $^{d}$ & 4 & $(3,7)$ & 2 & $(1,9)$ & n.a. & \multirow[t]{3}{*}{$1: 1^{f}$} & $P S$ \\
\hline Lumbald $^{d}$ & 39 & $(36,1)$ & 40 & $(37,0)$ & 0,91 & & $P S$ \\
\hline "Missing" & 4 & $(3,7)$ & 5 & $(4,6)$ & n.a. & & $P S$ \\
\hline \multicolumn{8}{|l|}{ Rückenschmerzdiagnosegruppe 2 im Vorjahr ${ }^{b, c}$} \\
\hline Spezifischer Rückenschmerz & 97 & $(89,8)$ & 95 & $(88,0)$ & 0,89 & - & PS \\
\hline Unspezifischer Rückenschmerz & 9 & $(8,3)$ & 9 & $(8,3)$ & 1,00 & - & PS \\
\hline "Missing" & 2 & $(1,9)$ & 4 & $(3,7)$ & n.a. & - & PS \\
\hline \multicolumn{8}{|c|}{ Rückenschmerzbezogene Leistungen regelhaft im Vorjahr ${ }^{b}$} \\
\hline Rücken-OP Vorvorjahr & 4 & $(3,7)$ & 4 & $(3,7)$ & n.a. & $1: 1$ & $P S$ \\
\hline Rücken-OP Vorjahr & 5 & $(4,6)$ & 5 & $(4,6)$ & 1,00 & $1: 1$ & $P S$ \\
\hline $\begin{array}{l}\text { Krankenhaus mit rückenbez. Hauptdiagnose } \\
\text { ohne ROP }\end{array}$ & 21 & $(19,4)$ & 15 & $(13,9)$ & 0,32 & - & PS \\
\hline Bilddiagnostik & 43 & $(39,8)$ & 48 & $(44,4)$ & 0,60 & - & PS \\
\hline Invasive Verfahren & 22 & $(20,4)$ & 18 & $(16,7)$ & 0,53 & - & PS \\
\hline Konservative Verfahren & 74 & $(68,5)$ & 72 & $(66,7)$ & 0,87 & - & PS \\
\hline Schmerzmittelverordnungen & 100 & $(92,6)$ & 100 & $(92,6)$ & 1,00 & $1: 1$ & $P S$ \\
\hline Physiotherapie & 70 & $(64,8)$ & 63 & $(58,3)$ & 0,54 & - & PS \\
\hline Facharztkontakt Rückenschmerz & 101 & $(93,5)$ & 104 & $(96,3)$ & 0,83 & - & PS \\
\hline Hilfsmittel Rückenschmerz & 34 & $(31,5)$ & 31 & $(28,7)$ & 0,71 & \multicolumn{2}{|l|}{ Kein Abgleich } \\
\hline Stationäre Anschlussrehabilitation & 3 & $(2,8)$ & 1 & $(0,9)$ & n.a. & - & PS \\
\hline Ambulante Rehabilitationsleistungen & 11 & $(10,2)$ & 6 & $(5,6)$ & 0,23 & - & PS \\
\hline
\end{tabular}




\begin{tabular}{|c|c|c|c|c|c|c|c|}
\hline \multirow[b]{2}{*}{ Merkmale } & \multicolumn{2}{|c|}{$\begin{array}{l}\text { Interventionsgruppe } \\
(\mathrm{IG}, N=108)\end{array}$} & \multicolumn{2}{|c|}{$\begin{array}{l}\text { Vergleichsgruppe } \\
\text { (VG, } N=108)\end{array}$} & \multirow{2}{*}{$\begin{array}{l}\begin{array}{l}\text { Unter- } \\
\text { schied } \\
\text { IG vs. VG }\end{array} \\
p \text {-Wert }\end{array}$} & \multicolumn{2}{|l|}{ Merkmalsabgleich } \\
\hline & $n$ & $\%$ & $n$ & $\%$ & & Ziehungsschritt 1 & Ziehungsschritt 2 \\
\hline \multicolumn{8}{|l|}{ Psychiatrische Diagnosen im Vorjahrb, ${ }^{b, c}$} \\
\hline Sucht (F1 ICD-10) & 9 & $(8,3)$ & 11 & $(10,2)$ & 0,65 & - & PS \\
\hline Schizophrenie (F2 ICD-10) & 1 & $(0,9)$ & 2 & $(1,9)$ & n.a. & - & PS \\
\hline Depression (F3 ICD-10) & 46 & $(42,6)$ & 38 & $(35,2)$ & 0,38 & - & PS \\
\hline Anhaltende Schmerzstörung (F454 ICD-10) & 30 & $(27,8)$ & 28 & $(25,9)$ & 0,79 & - & PS \\
\hline Sonstige Diagnosen F ICD-10 & 54 & $(50,0)$ & 44 & $(40,7)$ & 0,31 & - & PS \\
\hline Sonstiger Krankenhausaufenthalt ${ }^{b}$ & 34 & $(31,5)$ & 39 & $(36,1)$ & 0,56 & - & PS \\
\hline $\begin{array}{l}\text { Anzahl diverse ATC-3-Steller Vorjahr } \emptyset \text { (SD) [Me- } \\
\text { dian] }\end{array}$ & \multicolumn{2}{|c|}{$6,7(3,5)[7]$} & \multicolumn{2}{|c|}{$6,0(3,3)[5]$} & 0,26 & - & PS \\
\hline \multicolumn{8}{|l|}{ Arbeitsunfähigkeit } \\
\hline Arbeitsunfähig bei Beobachtungsbeginn & 18 & $(16,7)$ & 18 & $(16,7)$ & 1,00 & $1: 1$ & PS \\
\hline Krankengeldbezug bei Beobachtungsbeginn & 11 & $(10,2)$ & 12 & $(11,1)$ & 0,83 & \multicolumn{2}{|l|}{ Kein Abgleich } \\
\hline Krankengeldanspruch vor Beobachtungsbeginn & 34 & $(31,5)$ & 33 & $(30,6)$ & 0,90 & \multicolumn{2}{|l|}{ Kein Abgleich } \\
\hline \multicolumn{8}{|c|}{ Fakultative Ausschlusskriterien für Programmteilnehmer im Vorjahrb } \\
\hline Wirbelfrakturen & 1 & $(1,9)$ & 5 & $(4,6)$ & n.a. & \multicolumn{2}{|l|}{ Kein Abgleich } \\
\hline Parese & 0 & $(0,0)$ & 0 & $(0,0)$ & n.a. & \multicolumn{2}{|l|}{ Kein Abgleich } \\
\hline Myasthenie & 1 & $(0,9)$ & 0 & $(0,0)$ & 0,32 & \multicolumn{2}{|l|}{ Kein Abgleich } \\
\hline Krebs & 10 & $(9,3)$ & 19 & $(17,6)$ & 0,09 & \multicolumn{2}{|l|}{ Kein Abgleich } \\
\hline Wirbelsäulentumoren & 0 & $(0,0)$ & 0 & $(0,0)$ & n.a. & \multicolumn{2}{|l|}{ Kein Abgleich } \\
\hline Rentenantrag & 4 & $(3,7)$ & 4 & $(3,7)$ & n.a. & \multicolumn{2}{|l|}{ Kein Abgleich } \\
\hline „Propensity score“ $\varnothing(S D)$ [Median] & \multicolumn{2}{|c|}{$0,19(0,11)[0,17]$} & \multicolumn{2}{|c|}{$0,17(0,07)[0,16]$} & 0,26 & \multicolumn{2}{|l|}{ n.a. } \\
\hline \multicolumn{8}{|c|}{ 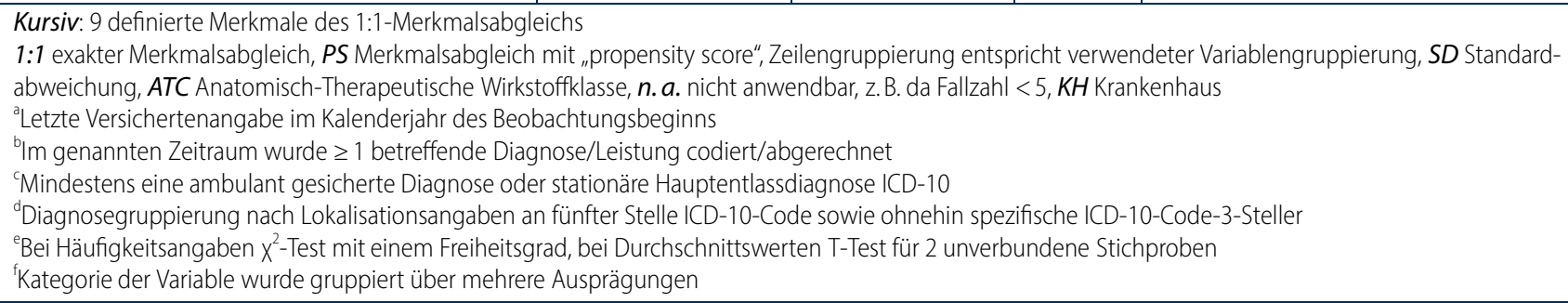 } \\
\hline
\end{tabular}

tete Hypothese geprüft, dass über den Vertrag RückenSPEZIAL die RBK reduziert werden. Die Analyse erfolgte mit SAS Enterprise Guide V6.1. Das "bootstrapping" erfolgte mit "proc survey select" und Ziehen mit Zurücklegen. Als Signifikanzniveau galt $a \leq 0,05$.

\section{Ergebnisse}

\section{Populationsbeschreibung}

Aus 135 Patienten der bereinigten Population der Interventionsgruppe konnte für 108 IG-Patienten $(80 \%)$ jeweils ein merkmalsgleicher VG-Patient identifiziert werden (- Abb. 1). Es wurden 108 IG-Patienten und 108 VG-Patienten verglichen. Aufgrund des exakten Merkmalsabgleichs waren jeweils 55,6\% weiblich. Das Durch- schnittsalter betrug 61 Jahre. 53,7\% hatten vor dem Indexquartal für mindestens 8 Quartale kontinuierlich in jedem Quartal eine codierte Rückenschmerzdiagnose. $4,6 \%$ hatten bereits mindestens eine ROP im Vorjahr (- Tab. 2).

Neben diesen ausgewählten exakt abgeglichenen Merkmalen hatten im Vorjahr 93,5\% der IG-Patienten mindestens einmal eine rückenschmerzbezogene ambulante Facharztbehandlung (VG 96,3\%), 64,8\% mindestens eine Physiotherapieleistung (VG 58,3\%), 20,4\% mindestens eine invasive Schmerztherapie/periradikuläre Therapie (VG 16,7\%), 19,4\% mindestens einen Krankenhausaufenthalt mit Hauptentlassdiagnose Rückenschmerz ohne ROP (VG 13,9\%, - Tab. 2).

Bei IG-Patienten erfolgten die Vorbefundprüfung und die IZMV im Median 6 Ta- ge nach dem Servicecenterkontakt (Durchschnitt 6,18 Tage, SD 13,90 Tage). In dieser Zwischenzeit fand regelhaft keine ROP statt.

\section{Weniger Patienten mit Rückenoperation}

In 365 Folgetagen hatten 47 IG-Patienten $(43,5 \%)$ und 81 VG-Patienten $(75,0 \%)$, also $42,0 \%$ weniger IG-Patienten, mindestens eine ROP (relatives Risiko [RR] 0,58; $p<0,001)$. Eine Betrachtung in drei Subgruppen zeigte:

- 20 IG-Patienten (18,5\%) erhielten beim IZMV eine direkte OP-Empfehlung.

- 21 IG-Patienten (19,4\%) erhielten zusätzlich zum IZMV auf ärztliche Empfehlung eine IMST. Von diesen IG-Patienten hatten $86,7 \%$ weniger 


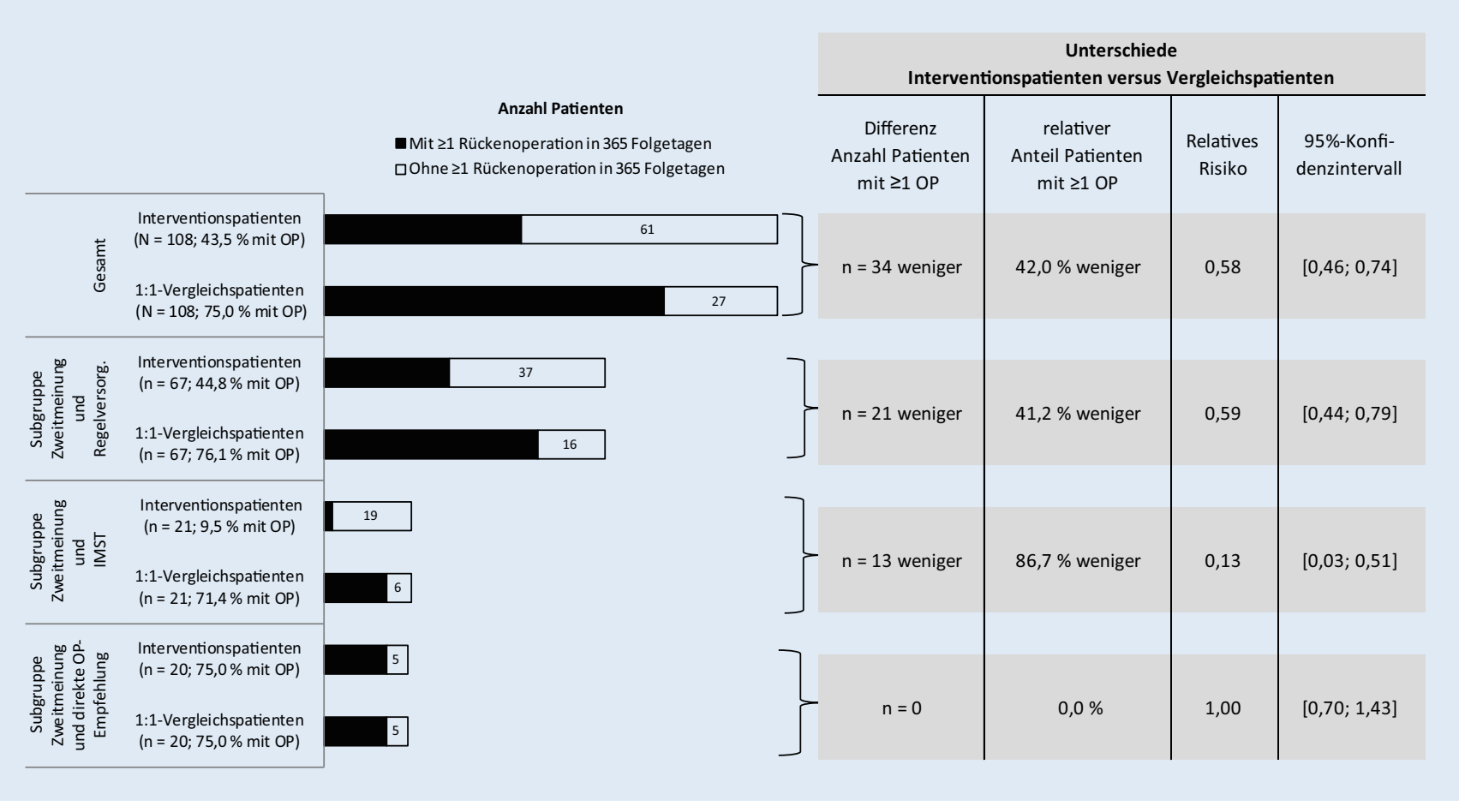

Abb. 2 \ Unterschiede bei Rückenoperationen in 365 Folgetagen. IMST Interdisziplinär-multimodale Schmerztherapie

mindestens eine ROP, im Vergleich zu ihren individuell merkmalsgleichen VG-Patienten (RR 0,13; $p<0,001$, $n=21)$.

- 67 IG-Patienten (62,0\%) erhielten „alleinig" IZMV. Von diesen IG-Patienten hatten $41,2 \%$ weniger mindestens eine ROP im Vergleich zu ihren individuell merkmalsgleichen VG-Patienten (RR 0,59; $p<0,001 ; n=67, \bullet$ Abb. 2).

\section{Weniger rückenschmerzbezogene Kosten}

Bei IG-Patienten stiegen die RBK - einschließlich der zusätzlichen Interventionskosten für den Vertrag RückenSPEZIAL um durchschnittlich 50,2\%-Punkte weniger stark an als bei den VG-Patienten $(p=0,088)$. Dieser positive Effekt war noch stärker ausgeprägt in der Subgruppe der IG-Patienten, die nach IZMV auf ärztliche Empfehlung eine kostenintensive IMST erhielten $(n=21$; durchschnittlich 115,0\%-Punkte niedrigerer Anstieg der RBK; $p=0,049)$. Patienten mit alleinigem IZMV hatten einen durchschnittlich 79,6\%-Punkte niedrigeren Anstieg der RBK gegenüber ihren individuell merkmalsgleichen VG-Patienten $(n=67 ; p=0,042$,
- Abb. 3). Die Unterschiede der RBK waren in den genannten Subgruppen signifikant, für die Gesamtgruppe allerdings knapp nicht.

Der insgesamt niedrigere Anstieg der RBK ging im Wesentlichen zurück auf anteilig weniger IG-Patienten mit Krankenhauskosten für ROP im Folgejahr. IG-Patienten hatten allerdings in den Leistungsbereichen Krankengeld, Schmerzmittel, Facharztbehandlung und Physiotherapie durchschnittlich einen geringfügig höheren Anstieg der RBK als die VG-Patienten (• Abb. 4).

\section{Diskussion}

Basierend auf einer Grundgesamtheit von Versicherten mit ROP-Erstmeinung und Krankenhauseinweisungsschein, identifiziert in den AOK-Servicecentern, erfolgte eine Matched-pairs-Analyse mit GKV-Daten. Vergleichsversicherte wurden aus dieser Grundgesamtheit gewonnen, die trotz Angebot durch die Krankenkasse nicht an dem Vertrag RückenSPEZIAL teilgenommen hatten. Die Analyse zeigte bei RückenSPEZIAL-Teilnehmern 42,0\% weniger Patienten mit mindestens einer Rückenoperation und 50,2\%-Punkte we- niger Anstieg rückenschmerzbezogener Kosten im Folgejahr.

\section{Weniger Patienten mit Rückenoperation}

Einarmige Fallbeobachtungen aus den USA berichteten aus ärztlichen Zweitmeinungseinschätzungen eine theoretische ROP-Vermeidbarkeit von 17,2\% [4], 44,5\% [6] oder $60,7 \%$ [3]. In Deutschland wurde eine ROP-Ablehnquote von $92,3 \%$ bei Zweitmeinungen zu bereits terminierten Operationen berichtet [13].

Bei 5 von 20 IG-Patienten mit ROPBestätigung im IZMV erfolgte in 365 Folgetagen keine ROP (• Abb. 2, Subgruppe Zweitmeinung und direkte OP-Empfehlung). Selbst bei ROP-Bestätigung im aufwendigen Zweitmeinungsverfahren entschied sich ein relevanter Anteil von Patienten im Nachgang dennoch gegen eine ROP. Eine ROP-Bestätigung oder -Ablehnung am Zweitmeinungstag ist scheinbar nur ein kurzfristig gültiger Endpunkt.

Ein belastbarerer Endpunkt ist eine datenbasiert vollständige Analyse von ROP für 365 Folgetage. Das wurde in Deutschland bereits mit GKV-Daten untersucht: Nach 92,3\% OP-Ablehnungen am Zweit- 


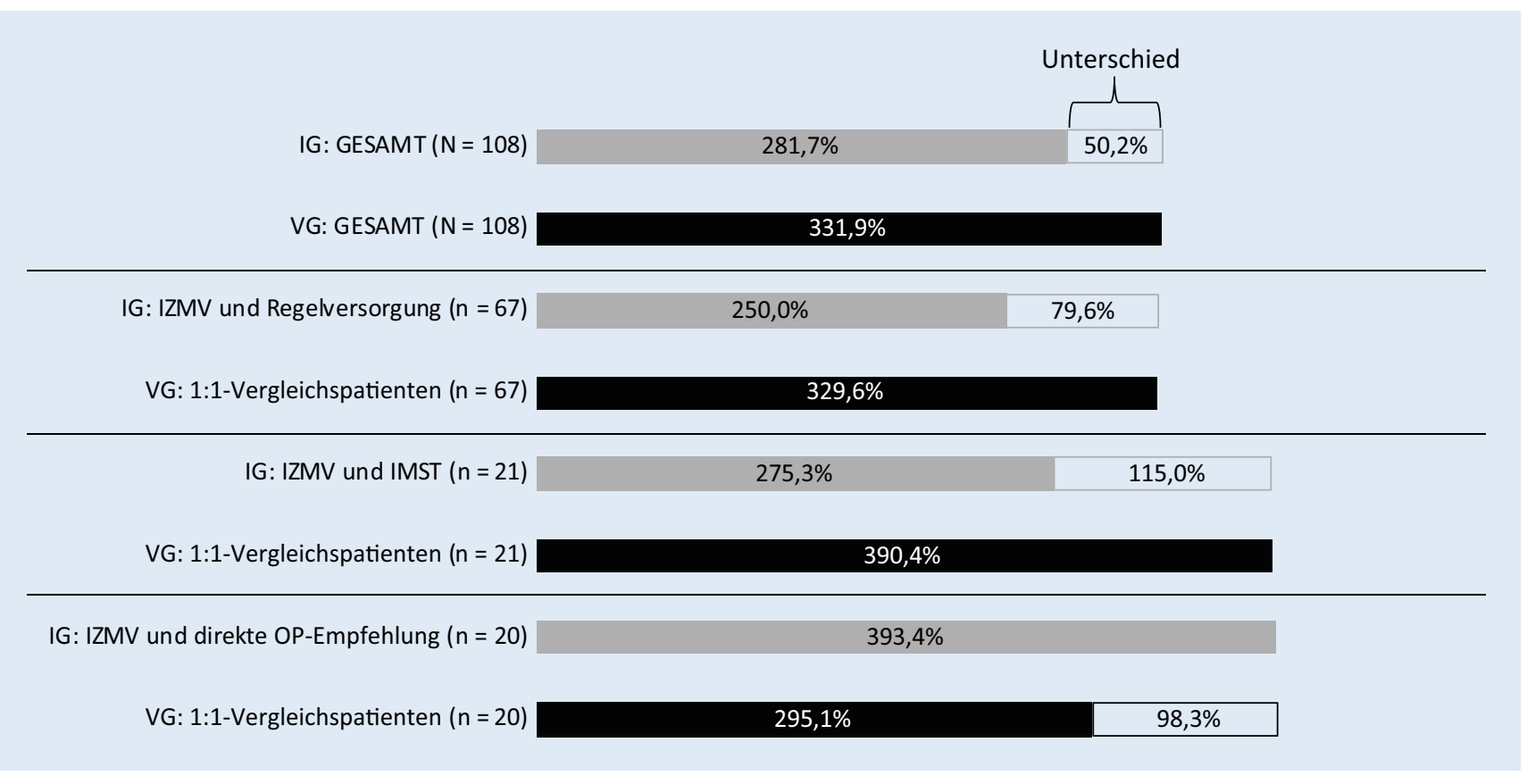

Abb. 3 ॥ Unterschiede im durchschnittlichen Anstieg rückenschmerzbezogener Kosten von Vorjahr auf Folgejahr (\%). Berechnung in Relation zu den Vorjahreskosten der Interventionsgruppe einschließlich der Kosten für RückenSPEZIAL; Zahlen aufgrund Rundung ggf. nicht summentreu. IG Interventionsgruppe; VG Vergleichsgruppe; IZMV interdisziplinäres Zweitmeinungsverfahren; IMST interdisziplinär-multimodale Schmerztherapie

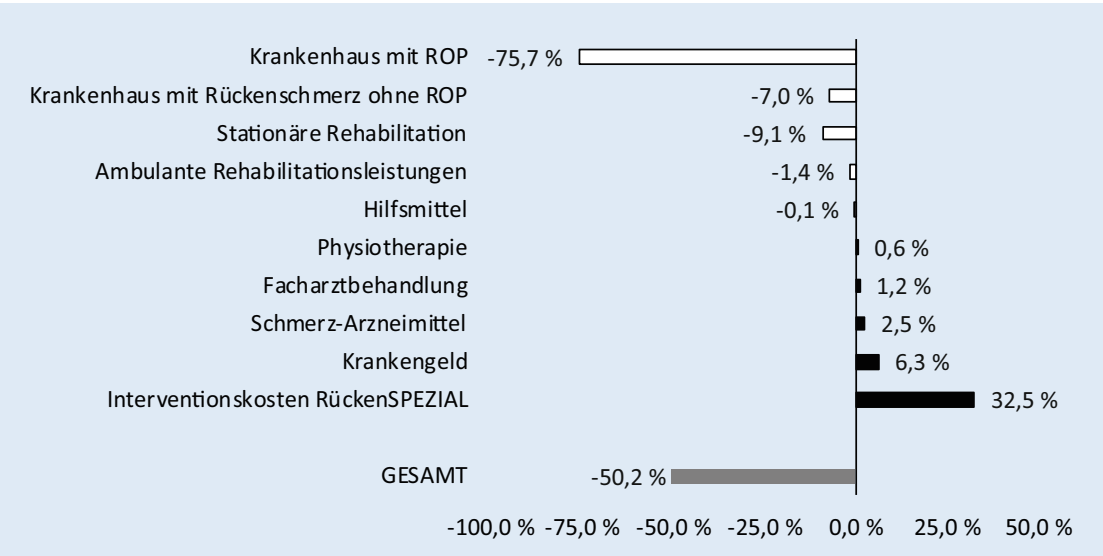

Abb. 4 \ Unterschiede im durchschnittlichen Anstieg rückenschmerzbezogener Kosten von Vorjahr auf Folgejahr je Leistungsbereich in \%-Punkten (Interventionsgruppe minus Vergleichsgruppe). Berechnung in Relation zu den Vorjahreskosten der Interventionsgruppe. ROP Rückenoperation

meinungstag erfolgte bei $79,0 \%$ der Zweitmeinungsteilnehmer in 12 Folgemonaten dauerhaft keine ROP [13]. Die vorliegende Studie ermittelte analog: Nach $81,5 \%$ OP-Ablehnungen erfolgte bei $56,5 \%$ der Zweitmeinungsteilnehmer in 12 Folgemonaten dauerhaft keine ROP (• Abb. 2, Gesamt 100,0-43,5\%). Der Unterschied bei ROP-Ablehnungen und dauerhafter ROPVermeidung in dieser Studie kann vermutlich mit einem 6 Jahre höheren Populationsdurchschnittsalter, ggf. entsprechend längerer Schmerzchronifizierung, abweichender Versichertenstruktur einer anderen Krankenkasse und ggf. auch mit abweichender Interventionsintensität erklärt werden.

Diese Studie verwendete erstmalig in Deutschland eine Matched-pairs-Vergleichsgruppe und zeigte weiterhin: Auch 25,0\% der Vergleichspatienten hatten in 12 Folgemonaten nach Ausstellung eines Krankenhauseinweisungsscheins keine ROP. Es ist möglicherweise denkbar, dass in einigen dieser Fälle vor der Ausstellung eines Krankenhauseinweisungsscheins für eine teure risikobehaftete ROP weitere alternative Maßnahmen erwägbar gewesen wären.

Mit der Intervention RückenSPEZIAL war eine Wirksamkeit von 42,0\% weniger $R O P$ als in der Vergleichsgruppe assoziiert. Eine Betrachtung alleinig der Zweitmeinungsteilnehmer mit 56,5\% (- Abb. 2, Gesamt 100,0-43,5\%) oder $79,0 \%$ [13] dauerhaft ausgebliebenen ROP kann zur Überschätzung der Wirksamkeit eines Zweitmeinungsverfahrens verleiten, wenn die Vergleichspatienten nicht beachtet werden, die auch ohne Zweitmeinungsverfahren dauerhaft keine ROP hatten.

Es zeigte sich ein Hinweis, dass eine vergleichsweise ROP-Vermeidung bei IGPatienten mit "IZMV und IMST" höher ausfällt als bei Patienten mit „IZMV und Regelversorgung". Für IMST wurden bereits positive Effekte auf die Wiederherstellung der Arbeitsfähigkeit ermittelt [15]. Die vorliegende Evaluation zeigte nun für IMST eine mögliche positive Assoziation mit ROPVermeidung. Diese Assoziation ist jedoch zumindest anteilig auch darauf zurückzuführen, dass Ärzte per IZMV-Empfehlung 
IMST-geeignete Patienten auswählten. Offenbar gibt es spezifisch geeignete Versicherte, bei denen IMST eine ROP abwenden kann. Eine nähere Beschreibung spezifischer Eignungskriterien könnte eine frühzeitige Identifikation dieser Patienten unterstützen.

\section{Kosteneffizienz dank hoher ROP- Wahrscheinlichkeit}

Für die Intervention des Vertrags RückenSPEZIAL gibt es aus GKV-Perspektive einen Hinweis auf Kosteneffektivität hinsichtlich der RBK, auch wenn das definierte Signifikanzniveau nicht erreicht wurde. Aufgrund der stringent gewählten Aufgreifkriterien gab es in der Studienpopulation eine hohe Wahrscheinlichkeit für ein tatsächlich zukünftig eintretendes, teures ROP-Ereignis. Damit konnte die Refinanzierung der zusätzlichen Interventionskosten des Vertrags RückenSPEZIAL maßgeblich durch ROP-Vermeidung gelingen. Studien zu Kosteneffekten für ein Zweitmeinungsverfahren bei ROP und für RBK konnten nicht gefunden werden [11]. Laut einer Studie aus dem Jahr 1982 auf Basis US-amerikanischer Abrechnungsdaten waren jedoch Zweitmeinungen bei einigen anderen elektiven Operationen kosteneffektiv [10].

Seit dem 19.11.2021 hat jeder gesetzlich Versicherte nach ärztlicher Indikationsstellung eines Eingriffs an der Wirbelsäule Anspruch auf ein Zweitmeinungsverfahren [5]. Beim besonderen Versorgungsprogramm RückenSPEZIAL wurden für die Wirksamkeit bei dauerhafter ROPVermeidung oder Kosteneffizienz zwei Erfolgsfaktoren deutlich, die für die konkrete Umsetzung oder Weiterentwicklung des generellen gesetzlichen Anspruchs auf ein Zweitmeinungsverfahren informativ und relevant sein können:

(1) Das Aufgreifkriterium für Zweitmeinung fokussierte auf einen eingegrenzten Kreis von Patienten, die bereits begonnen hatten, ihre ROP konkret organisatorisch vorzubereiten (Vorlage des Krankenhauseinweisungsscheins im AOK-Servicecenter). Das gewährleistete eine hohe ROPEintritts-Wahrscheinlichkeit und damit eine hohe Chance auf ROP-Vermeidung. (2) Das Zweitmeinungsverfahren erfolgte stets fachübergreifend und leitete direkt in fachübergreifend ermittelte konservative Behandlungsalternativen über bzw. leitete bei festgestellter Eignung direkt die umfangreiche konservative Maßnahme IMST ein.

\section{Stärken}

Zu den Stärken dieser GKV-Daten-Analyse zählt, dass sie teils erstmalig in Deutschland

- systematisch auf Daten zu ROP-Erstmeinungen zurückgreifen konnte,

- Rückenoperationen vollständig datenbasiert für 365 Tage nachbeobachtete,

- die Programmwirksamkeit mittels Matched-pairs-Vergleichsgruppe untersuchte und hinsichtlich ROP„Vermeidung" transparent machte, dass auch Vergleichspatienten teils keine ROP hatten,

- spezifisch rückenschmerzbezogene Merkmale und Kosten analysierte,

- eine stichtagsbezogene tagesgenaue Datenanalyse realisierte.

Die Analyse krankheitsspezifischer Kosten ermöglichte eine Verringerung von Verzerrungen durch möglicherweise nicht krankheitsassoziierte Kosten bspw. für eine Herzerkrankung.

\section{Limitationen}

Vergleichsversicherte wurden aus einer Grundgesamtheit von Patienten mit ROPKrankenhauseinweisungsschein gewonnen, die trotz des beratenden Angebots durch die Kasse nicht an dem Vertrag RückenSPEZIAL teilgenommen hatten. Entsprechend ist von einem Selbstselektionseffekt auch bei der Vergleichsgruppe auszugehen. In der Versorgungsrealität entscheidet sich der Patient für oder gegen eine Teilnahme am Vertrag RückenSPEZIAL und nicht eine Randomisierung. Diese Selbstselektion - RückenSPEZIAL-Teilnehmer hatten vermutlich eine erheblich kritischere Einstellung zur ROP - ist damit ein nicht näher differenzierbarer Bestandteil des unter realen Versorgungsbedingungen ermittelten Effekts.

Der Selbstselektionseffekt kann mit den vorliegenden Daten nicht weiter adjustiert werden. Die Daten ermöglichten jedoch grundlegend ein detailliertes „matching" rückenschmerzbezogener Merkmale. Allerdings konnten nicht alle datenverfügbaren Merkmale mit der Matched-pairsMethode bestmöglich adjustiert werden. Für drei Merkmale verblieben erkennbare Häufigkeitsunterschiede zwischen IG und VG (•Tab. 2):

1. IG-Patienten hatten häufiger einen rückenschmerzbezogenen Krankenhausaufenthalt ohne ROP im Vorjahr.

2. IG-Patienten hatten häufiger Depressionsdiagnosen.

3. IG-Patienten hatten seltener nichtwirbelsäulenassoziierte Krebsdiagnosen.

In der Gesamtschau dieser und weiterer geringfügiger Häufigkeitsunterschiede wird angenommen, dass daraus resultierende Verzerrungen oder mögliche Über-/ Unterschätzungen des Effekts sich tendenziell gegenseitig ausbalancieren. Weitere Ergebnisverzerrungen können nicht ausgeschlossen werden, falls relevante, aber nicht (vollständig) verfügbare Merkmale wie etwa die Schmerzklassifikation nach von Korff, Sportaktivität oder Beruf ungleich verteilt sind. Weiterhin können bislang unbekannte Störgrößen die Effektschätzer verzerren.

\section{Fazit für die Praxis}

- Mit besonderen Dokumentationsdaten zu AOK-Beratungen konnten Erstempfehlungen zu Rückenoperationen einheitlich erfasst werden. Dies ermöglichte - mit den genannten Limitationen - die erste kontrollierte Interventionsstudie in Deutschland für ein Versorgungsprogramm mit Zweitmeinungsverfahren bei empfohlenen Rückenoperationen.

- Bei Patienten, die sich im realen Versorgungsalltag für eine Teilnahme am Zweitmeinungsverfahren entschieden, hatten im Folgejahr $42 \%$ weniger eine Rückenoperation als bei Vergleichspatienten, die sich gegen eine Teilnahme entschieden.

- Patienten, die nach der Zweitmeinung auf ärztliche Empfehlung noch intensive Betreuung mit interdisziplinär-multimodaler Schmerztherapie erhielten, hatten den geringsten Anteil an Rückenoperationen im Folgejahr.

- Aus Perspektive der gesetzlichen Krankenversicherung erscheint das Versorgungsprogramm RückenSPEZIAL kosteneffizient, maßgeblich aufgrund von Kosteneinsparungen bei Rückenoperationen.

- Das Programm ist praxistauglich sowohl mit als auch ohne zusätzliche interdiszi- 
plinär-multimodale Schmerztherapie für eine spezifische Population mit bereits ausgestelltem Krankenhauseinweisungsschein für eine Rückenoperation.

\section{Korrespondenzadresse}

Dr. Christoph J. Wagner

AOK Nordost

Wilhelmstraße 1, 10963 Berlin, Deutschland christoph.wagner@nordost.aok.de

Danksagung. Wir danken Dr. Katrin Reber und Dr. Sebastian Liersch von der AOK Nordost ausdrücklich für die inhaltliche und sprachliche Prüfung des Manuskripts.

\section{Einhaltung ethischer Richtlinien}

Interessenkonflikt. C.J. Wagner und S. Sehlen gewährleisten für die AOK Nordost das Controlling und die Evaluation des Vertrags RückenSPEZIAL. G. Ayyad, A. Otzdorff und W. Wyrwich gewährleisten für die AOK Nordost die Entwicklung und organisatorische Betreuung des Vertrags RückenSPEZIAL. U. Marnitz, K. Bienek und B. von Pickardt sind Vertragspartner und erbringen die Versorgungsleistung des Vertrags RückenSPEZIAL. G. Lindena kooperiert zur Dokumentation und Evaluation der RückenSPEZIAL-Versorgung.

Für diesen Beitrag wurden von den Autoren keine Studien an Menschen oder Tieren durchgeführt. Für die aufgeführte Sekundärdatenanalyse gelten die jeweils dort angegebenen ethischen Richtlinien.

Open Access. Dieser Artikel wird unter der Creative Commons Namensnennung 4.0 International Lizenz veröffentlicht, welche die Nutzung, Vervielfältigung, Bearbeitung, Verbreitung und Wiedergabe in jeglichem Medium und Format erlaubt, sofern Sie den/die ursprünglichen Autor(en) und die Quelle ordnungsgemäß nennen, einen Link zur Creative Commons Lizenz beifügen und angeben, ob Änderungen vorgenommen wurden.

Die in diesem Artikel enthaltenen Bilder und sonstiges Drittmaterial unterliegen ebenfalls der genannten Creative Commons Lizenz, sofern sich aus der Abbildungslegende nichts anderes ergibt. Sofern das betreffende Material nicht unter der genannten Creative Commons Lizenz steht und die betreffende Handlung nicht nach gesetzlichen Vorschriften erlaubt ist, ist für die oben aufgeführten Weiterverwendungen des Materials die Einwilligung des jeweiligen Rechteinhabers einzuholen.

Weitere Details zur Lizenz entnehmen Sie bitte der Lizenzinformation auf http://creativecommons.org/ licenses/by/4.0/deed.de.

\section{Literatur}

1. Ali J, Pieper D (2017) Kaum aktuelle Daten zu Zweitmeinungsverfahren vorhanden - eine systematische Ubersichtsarbeit. Gesundheitswesen 79:871-874

Less surgery and lower cost due to back pain in a care program with an interdisciplinary second opinion procedure. A controlled nonrandomized intervention study based on claims data

Background: There is no evidence of effectiveness for interdisciplinary second opinion procedures (ISOP) for recommended back surgery (BS). Since 2015, AOK Nordost has been offering the care program RückenSPEZIAL comprising a preliminary examination, ISOP, and optional interdisciplinary multimodal pain therapy (IMPT). The objective of this study is to determine the effectiveness of RückenSPEZIAL to reduce $\mathrm{BS}$ and back pain-related costs (BPRC) compared to patients who likewise received a recommendation for back surgery but not RückenSPEZIAL.

Methods: Insured persons in the AOK Nordost consulted the AOK service center, presented a BS hospital admission slip and received advice to participate in RückenSPEZIAL. Following a 1:1 "matched pairs" selection, patients who participated in RückenSPEZIAL (intervention group [IG]) after this consultation (reference date) where compared with patients who did not participate after this consultation (comparison group [CG]). Patient characteristics, BS and BPRC were operationalised from AOK Nordost claims data.

Results: Of 108 IG patients and 108 CG patients, 34 (42\%) fewer IG patients had one or more $\mathrm{BS}$ in 365 following days (relative risk [RR] 0.58; $p<0.001$ ). The subgroup analysis showed for 21 IG patients with ISOP and IMPT an RR of $0.13(p<0.001)$, and for 67 IG patients with solely ISOP without IMPT an RR of $0.59(p<0.001)$. The increase in RBC from the previous year to the following year was 50.2 percentage points lower for IG patients compared to CG patients ( $p=0.088)$.

Discussion: The differences in BS were significant $(p<0.05)$ and in favor of RückenSPEZIAL. For the specific population it can be expected that mainly savings on BS can cover the intervention costs of RückenSPEZIAL (approximately significant, small case number). Bias due to self-selection needs to be assumed.

\section{Keywords}

Elective surgery $\cdot$ Spine surgery $\cdot$ Chronic back pain · Pain therapy · Cost of illness

2. Destatis (2019) Fallpauschalenbezogene Krankenhausstatistik (DRG-Statistik) Operationen und Prozeduren der vollstationären Patientinnen und Patienten in Krankenhäusern (4 Steller) 2018. https://www.destatis.de. Zugegriffen: 12. Okt. 2021

3. Epstein NE (2013) Are recommended spine operations either unnecessary or too complex? Evidence from second opinions. Surg Neurol Int 4:353-358

4. Epstein NE, Hood DC (2011) "Unnecessary" spinal surgery: a prospective 1-year study of one surgeon's experience. Surg Neurol Int 2:83

5. G-BA (2021) Beschluss des Gemeinsamen Bundesausschusses über eine Änderung der Richtlinie zum Zweitmeinungsverfahren ( $\mathrm{Zm}-\mathrm{RL}$ ): Aufnahme von Eingriffen an der Wirbelsäule in den Besonderen Teil der Richtlinie. Bundesanzeiger AT 18.11.2021 B5. Bundesministerium für Gesundheit,

6. Gamache FW (2012) The value of "another" opinion for spinal surgery: a prospective 14-month study of one surgeon's experience. Surg Neurol Int 3:350-354

7. Marnitz U (2020) Die interdisziplinäre, multimodale Schmerztherapie in der konservativen Wirbelsäulentherapie. Wirbelsäule 04:168-173

8. Nürnberg V, Meier M-T (2021) Patientenrecht Zweitmeinung. Springer,

9. Pieper D, Hess S, Mathes T (2018) Bestandsaufnahme zu Zweitmeinungsverfahren in der
Gesetzlichen Krankenversicherung (GKV). Gesundheitswesen 80:859-863

10. Ruchlin HS, Finkel ML, McCarthy EG (1982) The efficacy of second-opinion consultation programs: a cost-benefit perspective. Med Care 20:3-20

11. S-V-R (2018) Sachverständigenrat zur Begutachtung der Entwicklung im Gesundheitswesen: Gutachten 2018, Bedarfsgerechete Steuerung der Gesundheitsversorgung. MWV, Berlin

12. Swart E, Bitzer EM, Gothe Het al (2016) A consensus German reporting standard for secondary data analyses, version 2 (STROSA-STandardisierte BerichtsROutine fuer SekundaerdatenAnalysen). Gesundheitswesen 78:e145-e160

13. Überall MA, Müller-Schwefe GH, Nolte Tetal (2020) Chronische Rückenschmerzen: operieren oder nicht? Schmerzmedizin 36:52-56

14. von Elm E, Altman DG, Egger M et al (2014) The strengthening the reporting of observational studies in epidemiology (STROBE) statement: guidelines for reporting observational studies. Int J Surg 12:1495-1499

15. WagnerCJ,AyyadG,OtzdorffAetal (2019) Behandlungs- und Kosteneffekte der interdisziplinaren multimodalen Schmerztherapie bei Patienten mit Ruckenschmerz: Eine kontrollierte, nichtrandomisierte Interventionsstudie mit GKV-Daten und Teilnehmerbefragung. Schmerz 33:128-138 
Hier steht eine Anzeige.

黑 Springer 